\title{
Le Linké Poalé Zion et la Résistance en Belgique durant la Seconde Guerre mondiale
}

Jeannine Levana Frenk

\section{OpenEdition}

12 Journals

Édition électronique

URL : http://journals.openedition.org/cmc/329

DOI : $10.4000 / \mathrm{cmc} .329$

ISSN : 2684-3080

Éditeur

Fondation de la Mémoire Contemporaine

Édition imprimée

Date de publication : 1 décembre 2016

Pagination : 63-98

ISSN : 1377-1256

\section{Référence électronique}

Jeannine Levana Frenk, « Le Linké Poalé Zion et la Résistance en Belgique durant la Seconde Guerre mondiale ", Les Cahiers de la Mémoire Contemporaine [En ligne], 12 | 2016, mis en ligne le 05 novembre 2019, consulté le 08 septembre 2020. URL : http://journals.openedition.org/cmc/329 ; DOI : https:// doi.org/10.4000/cmc.329 


\section{Le Linké Poalé Zion et la Résistance en Belgique durant la Seconde Guerre mondiale}

\section{Jeannine Levana Frenk}

L'historiographie n'a pas toujours été très généreuse avec le Linké Poalé Zion - LPZ (les Ouvriers de Zion de gauche) et sa contribution à la Résistance juive en Belgique durant la Seconde Guerre mondiale. Cet article propose une relecture de la question fondée sur l'analyse de sources nouvelles et de la presse clandestine du parti ${ }^{1}$.

Le LPZ fut créé en 1920 suite à une scission au sein de l'Union mondiale du Poalé Zion - PZ, le courant sioniste majoritaire, au cours de son congrès tenu à Vienne la même année. Dès avant la rupture de 1920, Dov-Ber Borochov, l'idéologue de la faction dissidente, accusait le $\mathrm{PZ}$ d'être sioniste à $85 \%$ et socialiste à seulement $15 \%$, alors que lui se voulait à la fois sioniste et socialiste à $100 \%$. Aspirant à combiner sionisme et révolution prolétarienne, l'exigence du groupe de Borochov de s'intégrer à la $\mathrm{III}^{\mathrm{e}}$ Internationale communiste mena à la rupture finale. Parti rebelle par excellence, le LPZ gravita pourtant, jusqu'à la fin des années 1930, en marge du mouvement communiste international parce qu'il revendiquait sa spécificité juive et défendait la cause sioniste, mais aussi des institutions sionistes mondiales, dont il s'était détaché. Cette dualité poussa Lénine, au cours d'une rencontre en Belgique avec Borochov, à définir ce qui allait devenir le LPZ comme un parti « assis entre deux chaises $»^{2}$. Ce caractère hybride lui a probablement permis de jouer les intermédiaires et de faire le lien entre communistes, sionistes généraux, Poalé Zion-Zéiré Zion (la jeune garde du Poalé Zion) et autres tendances, au moment de la création du Comi-

\footnotetext{
${ }^{1}$ Le présent article est le résultat de recherches menées essentiellement dans des fonds d'archives en Israël.

${ }^{2}$ Borochov, menacé d'arrestation à cause de son activité politique, dut fuir la Russie tsariste et trouva d'abord refuge en Belgique avant de poursuivre vers les États-Unis. Il s'installa à Liège, où vivait une "colonie" d'étudiants immigrés. Selon les mémoires de sa femme, Liova Borochov, la rencontre de son mari avec Lénine, venu à Liège pour y donner une conférence, eut lieu en 1907 ou 1908. Voir L. Borochov, Prakim Mé Yoman Hayaï, Guivat Haviva, 1971, pp. 24-25.
} 
té de Défense des Juifs en 1942. L’engagement du LPZ en défense des masses juives découla de sa tradition dissidente, forgée dans le dévouement sans bornes de ses militants et dans sa nature de parti "trait d'union" entre communistes et sionistes de toutes tendances.

\section{Le LPZ en Belgique de l'entre-deux-guerres}

Le LPZ a existé en Belgique de 1921 à 1948 quand il fut intégré au Mapam $^{3}$. Il était particulièrement bien implanté au cour de l'immigration yiddishophone originaire d'Europe de l'Est, venue s'installer dans le pays durant l'entre-deux-guerres. Il reproduisait au sein de la yiddishe gas (la rue juive) des microcosmes de vie calqués sur les structures politiques et culturelles du monde que les nouveaux arrivants venaient juste de quitter. L'historien Rudi Van Doorslaer a noté que ces cercles s'érigeaient en "famille de substitution qui constituait, dans un environnement étranger, un lieu de convivialité $»^{4}$ ou des « synagogues de substitution $\aleph^{5}$. Par ailleurs, le parti regroupait à la fois des intellectuels, ingénieurs et médecins férus de langue et de culture yiddish, et des ouvriers autodidactes, qui incarnaient ce rapport privilégié entre culture et radicalisme politique, caractéristique des révolutionnaires du yiddishland décrit par Alain Brossat et Sylvia Klingberg6. La plupart des ouvriers-intellectuels radicaux qui militaient au LPZ de Belgique combinaient en effet l'action politique et culturelle avec l'exercice d'un métier manuel, ce qui leur permit de maintenir des liens étroits avec les milieux ouvriers juifs.

En 1928, Bruxelles accueillit la première conférence des représentants du LPZ établis dans les pays d'Europe occidentale. La Belgique y était représentée par David/Dodé Trocki, qui terminait à ce moment ses études d'ingénieur en chimie à l'université de Gand, et par Yéhuda

\footnotetext{
${ }^{3}$ Le Mapam (Parti ouvrier unifié) fut créé en Palestine mandataire en janvier 1948. Il représentait l'aile gauche du parti travailliste (le Mapai) regroupé autour de David Ben Gourion. Le Mapam obtint 19 sièges de députés au Parlement aux premières élections générales tenues après la création de l'État d'Israël. Il constitua ainsi le second plus grand parti du pays. Abusz Werber créa l'antenne belge du nouveau parti.

${ }_{4}^{4}$ R. Van Doorslaer, Enfants du Ghetto. Juifs révolutionnaires en Belgique (1925-1940), Bruxelles, 1997, p. 66.

5 Ibid, p. 103.

6 A. Brossat - S. Klingberg, Le yiddishland révolutionnaire, Paris, 2009.
} 
Tiberg, journaliste et dirigeant du parti d'avant-guerre ${ }^{7}$. Un an plus tard, Abusz Werber, coupeur de chaussures et fervent " yiddishiste », quittait sa Pologne natale pour se joindre à l'équipe dirigeante du LPZ belge $^{8}$. En 1931, Trocki et Werber scellèrent leur alliance politique en devenant beaux-frères, faisant du parti une affaire de famille. D'autres personnes engagées à leurs côtés, tels les Tabakman et les Bunim, étaient également unies par des liens familiaux ${ }^{9}$.

Le LPZ était faiblement représenté à Anvers. À Bruxelles, par contre, il réussit à gagner une audience relativement importante dans le courant des années 1930. En 1939, il représentait $32 \%$ de l'électorat sioniste, un "phénomène remarquable », selon Van Doorslaer, vu la marginalité du LPZ et le fait qu'il n'intégra les institutions du judaïsme officiel, tels le Congrès Juif mondial et la Fédération sioniste de Belgique, qu'en 193710. Les deux partis combinés du LPZ et PZ donnèrent alors une impulsion sans précédent au sionisme ouvrier de Belgique qui devint prédominant dans la capitale. En 1939, ensemble, ils y recueillirent $56 \%$ des voix aux élections du Congrès juif mondial (48\% à Liège et $47 \%$ à Charleroi), contre $35 \%$ des voix à Anvers.

Plus importante encore fut la contribution du LPZ au Conseil des Associations juives de Bruxelles (Rat fun die Yiddishe Organizatsies in Brisl), créé en 1937, qui coalisait en un front unifié toutes les tendances actives dans la rue juive, réunissant vingt-sept organisations, des communistes aux orthodoxes. Ce Conseil se distingua dans la lutte contre la montée de l'antisémitisme, contre l'expulsion des sanspapiers et l'allègement de la législation contre les étrangers. Ainsi, « le Linké Poalé Zion était à l'avant-plan dans les activités du Conseil » et en était devenu l'une de ses forces les plus actives ${ }^{11}$. Yéhuda Tiberg représentait le LPZ au sein du comité exécutif du Conseil et était le rédacteur en chef de son journal hebdomadaire, Unzer Yichouv, publié

\footnotetext{
7 Sur la cellule des étudiants juifs affiliés au LPZ dans les instituts d'enseignement supérieur de Gand, voir R. Van Doorslaer, op. cit., p. 85.

8 Pour plus d'information sur les parcours de ses parents Abusz et Shifra Werber, de son oncle et sa tante, Dodé et Pauline Trocki, voir le livre publié par Michel Werber, La Parole d'Abusz Werber, Bruxelles, 2015.

9 Voir J.-Ph. Schreiber, Dictionnaire biographique des Juifs de Belgique. Figures du judaïsme belge XIXe-XXe siècles, Bruxelles, 2002, « Tabakman Szrul-Israël », p. 333.

${ }^{10}$ R. Van Doorslaer, op. cit., p. 38.

11 Ibid, p. 207.
} 
en yiddish, dès mars 193912. Abusz Werber apporta une contribution de poids à sa rédaction. Lui et Dodé Trocki dirigeaient la commission culturelle du Conseil et mirent sur pied L'Université populaire juive de Bruxelles où les cours étaient donnés en yiddish et en français. Ils y animaient la section yiddish. Werber y enseigna la sociologie juive et l'histoire du mouvement ouvrier, Trocki l'histoire juive. Shlomo Hochberg donna des cours de littérature yiddish et I. Austriak de littérature hébraïque. K. Motiuk et le docteur Socrate Helman couvrirent les domaines des sciences naturelles et de l'hygiène.

Le LPZ se tailla aussi une assise solide dans le champ de l'activité syndicale, en particulier au sein de la sous-section juive des ouvriers de la maroquinerie, affiliée au syndicat des peaux, cuirs et fourrures. Il est possible que cela soit lié à l'activité d'Abusz Werber, d'Israël (Srul) Tabakman et de son fils Meyer, ou des Bunim, qui géraient chacun leur propre atelier de fabrication de chaussures. Il s'agissait de petites fabriques familiales employant essentiellement un ouvrier-patron et une ou deux aides. Avraham Domb, un pilier du LPZ, dirigeait cette branche du syndicat avec Jacob Gutfraynd, d'obédience communiste. Il faut ajouter que le LPZ gérait aussi un club populaire, Folks Klab, une «École pour l'éducation », Shul un Derziung, un mouvement de jeunesse, le Yung Bor (diminutif de Borochov Yugent) et un club sportif, le Shtern, administré par Meyer Tabakman ${ }^{13}$.

\footnotetext{
12 Yéhuda Tiberg (1900-1984) avait étudié à l'université de Varsovie, où il avait fait partie du cercle des historiens regroupés autour d'Emanuel Ringelblum et de Raphael Mahler. Il s'enfuit de Pologne en 1923 suite à la vague de répression contre les militants de gauche. Après un court séjour à Danzig, il s'installa en Belgique en 1924 et contribua à la consolidation du LPZ. Journaliste de profession, il fut aussi le rédacteur en chef du journal yiddish Unzer Weg édité par le parti à New York, où il trouva refuge en 1940. Voir les articles nécrologiques publiés dans Y. Tiberg, Ayf di Schliachen fun Zayt, Tel Aviv, 1987 (en yiddish).

13 Voir D. Michman, «Les mouvements de jeunesse sionistes en Belgique durant l'occupation allemande. Étude d'un point de vue comparatif », dans R. Van Doorslaer (dir.), Les Juifs de Belgique. De l'immigration au génocide, 1925-1945, Bruxelles, 1994, pp. 173-192.
} 


\section{Pour un État juif indépendant ou pour le statut de minorité natio- nale?}

Si le LPZ se voulait sioniste et socialiste à $100 \%$, il préconisait tout d'abord l'émancipation des Juifs de l'étau du ghetto et le redressement de la « pyramide inversée ». Concept développé par Borochov, il visait à abolir l'anomalie de la condition économique particulière des Juifs en diaspora. Celle-ci était due à l'inexistence d'une classe rurale à sa base, remplacée par un prolétariat artisanal concentré dans le domaine de la production tertiaire (plutôt que dans l'industrie lourde) et en voie de paupérisation, surtout en période de crise, avec en son centre une large classe moyenne composée de commerçants et, à sa tête, une petite classe de capitalistes et d'intellectuels pratiquant les professions libérales. Certes, pour Borochov, la création d'un État souverain en Palestine permettrait de normaliser les structures économiques des communautés juives et de redresser la pyramide afin d'assurer l'établissement d'une société agricole, industrielle et égalitaire. Mais, avec la rupture de 1920 et le maintien du parti à l'écart des institutions sionistes jusqu'en 1937, le projet étatique fut renvoyé à un "futur" de caractère messianique. Le parti opta pour la priorité de la lutte de la classe ouvrière juive aux côtés du prolétariat international, dans le présent et sur les lieux de résidence des Juifs, de manière à faire advenir le socialisme dans le cadre de la révolution mondiale. L'assainissement des structures économiques particulières aux communautés juives se concevait ainsi à travers l'abolition future du régime capitaliste.

Les premiers numéros de Unzer Wort (Notre Parole), le journal clandestin du LPZ qu'Abusz Werber lança en décembre 1941, réaffirment en revanche la nécessité de la solution territoriale et la concentration du peuple juif en Palestine. Mais dans son numéro 4 de juin 1942, le Unzer Wort clandestin déclare encore que l'option palestinienne ne peut pas être l'unique solution aux problèmes des Juifs : « La Palestine ne peut pas constituer l'unique solution... parce que la Palestine ne peut pas accueillir tous les Juifs. Et des parties importantes du peuple seront obligées, par conséquent, de rester en diaspora où elles devront se battre pour obtenir des droits de minorité nationale. La Palestine deviendra donc l'État qui, selon une expression de Borochov, sera la force capable de protéger les droits de cette minorité na- 
tionale ${ }^{14}$. Du fait de la guerre, les Juifs restaient encore dispersés et les possibilités d'émigration étaient nulles, le LPZ n'avait donc pas encore fixé son choix : la lutte pour la création d'un État juif souverain et/ou l'exigence du statut de minorité nationale dans les pays de la diaspora.

Unzer Wort maintient jusqu'en 1943 cette position de caractère presque "autonomiste", proche du Bund («Union générale des travailleurs juifs de Lituanie, de Pologne et de Russie », mouvement socialiste juif non sioniste) jouant encore avec l'éventualité d'une existence juive en tant que minorité nationale dans les pays d'Europe, option importée de Pologne et quelque peu utopique dans le cas de la Belgique. Mais à partir de 1943, les informations sur les déportations et l'étendue de la destruction des communautés juives d'Europe qui parviennent aux rédacteurs mettent un terme à l'idée d'un maintien possible en tant que minorités nationales. Les convictions relatives à l'établissement d'un État juif en Palestine prennent alors le dessus au sein du LPZ.

Jusqu'en 1942-43, le LPZ resta de fait plus socialiste que sioniste, puisqu'il n'encouragea ni l'émigration de masse vers la Palestine ni la mobilisation de son mouvement de jeunesse en vue de l'avènement de l'État. Le Yung Bor se limita à un rôle de formation intellectuelle, contrairement à d'autres mouvements de jeunesse sionistes de gauche, tels l'Hashomer Hatzaïr ou le Dror, qui donnaient la priorité au « constructivisme pionnier », c'est-à-dire à la « montée » vers EretzIsrael, l'Alya, et à cet effet à la préparation des jeunes dans des centres de formation agricole, les hachsharot. Pour le LPZ en revanche, créer une avant-garde pionnière et sélective destinée à la conquête de la terre n'était pas encore à l'ordre du jour. Par contre, la défense des masses juives s'imposait en priorité là où elles se trouvaient : sans elle, il n’y aurait pas d'existence possible, ni sociale, ni culturelle et encore moins nationale ou territoriale.

Cette divergence entre le LPZ et les membres de l'Hashomer Hatzaïr, en l'occurrence, eut des répercussions directes sur leur engagement

\footnotetext{
14 Unzer Wort, 4, juin 1942, p. 3. Pour les originaux en yiddish du journal clandestin Unzer Wort, voir Archives Yad Vashem (YVS), 0.29.2/3727483. Cette collection comprend 18 numéros retrouvés. J'ai utilisé leur traduction en français de M. Freund, gracieusement mise à ma disposition par Michel Werber.
} 
dans la lutte contre l'occupant. En 1942, Werber voulut les fédérer dans la Résistance. Il se rendit à Anvers pour les convaincre de se joindre au mouvement clandestin. Mais ils rejetèrent sa proposition, lui expliquant que ce qui se passait en diaspora ne les intéressait pas. Ils préféraient quitter la Belgique pour rejoindre la Suisse et gagner EretzIsrael, ou, s’ils échouaient, rester en Suisse pour se préparer à émigrer au plus tôt ${ }^{15}$. Face à la priorité du sionisme pour le « constructivisme » pionnier, de caractère élitiste et sélectif, le LPZ se positionnait au contraire en faveur des masses prolétaires paupérisées. Abusz Werber s'opposa personnellement à cet effet à toute défection pendant la durée de l'Occupation : il fallait rester sur place et lutter aux côtés des persécutés, de ceux qui pour des raisons diverses n'avaient pas eu l'opportunité de fuir.

\section{Priorité au yiddish}

Un autre sujet de divergence entre le LPZ et le sionisme dominant concernait la langue. Le LPZ s'entêtait à préserver la langue du peuple, le yiddish, et sa culture, plutôt que de contribuer à la renaissance de la langue hébraïque, objectif primordial aux yeux des promoteurs du projet national. La préférence du LPZ pour le yiddish et sa culture, plutôt que pour l'hébreu des textes sacrés, découlait de son approche prolétarienne. Étant la langue du peuple, la mamé loushn (la langue maternelle) de l'immense majorité des ouvriers juifs de l'Europe de l'Est, il fallait la conserver pour toucher les masses juives, en tant que vecteur de diffusion du savoir et des idéaux socialistes. L'enseignement en yiddish représentait donc un aspect du travail politique en vue d'élever le niveau des ouvriers et développer leur conscience de classe. Le LPZ proposait aussi l'utilisation de cette langue populaire

15 Interview d'Abusz Werber, Université hébraïque de Jérusalem, Institut du Judaïsme contemporain, département de documentation orale, 04.03.1964, 2 ${ }^{\mathrm{e}}$ session. Sur l'Hashomer Hatzaïr en Belgique, voir l'excellent travail de fin d'études de Janiv Stamberger : Be Strong and Brave! A Small Youth Movement in a Sea of History. The Hashomer Hatzaïr in Antwerp (1920-1948), Université de Gand, 2012-2013. Je remercie Janiv Stamberger de m'avoir transmis son manuscrit. Voir aussi J. Stamberger, «Zionist Pioneers at the Shores of the Scheldt. The Hashomer Hatzaïr Youth Movement in Antwerp, 1924-1946 », dans Les Cahiers de la Mémoire contemporaine, 11, 2014, pp. 95-99. 
commune pour faire le lien entre les différentes communautés dispersées à travers le monde. D’autre part, dès la fin du XIXe siècle, le yiddish subit une transformation et un essor extraordinaire. D’un jargon populaire, il se modernisa et devint une langue littéraire ainsi que le fil conducteur d'une haute culture profane et läque en pleine expansion. C'est ce yiddish-là que les membres du LPZ s'acharnaient à promouvoir. Outre son implication dans l'école du parti, sa bibliothèque yiddish et la mise en place de l'Université populaire juive de Bruxelles, Trocki était le correspondant et le "Zamler" (collectionneur), pour la Belgique, du YIVO (Yiddisher Visnshaftlekher Institut), Institut de recherche juive fondé à Vilno en 1925. Il lui envoya des études sociologiques sur la communauté juive, dont une Histoire de la presse yiddish en Belgique, ainsi que divers matériaux puisés dans les archives du pays. Sa sœur Shifra, épouse d'Abusz Werber, écrivait des poèmes en yiddish et enseignait sa littérature. Tous deux, avec Pauline, l'épouse de Trocki, animaient un cercle de théâtre yiddish et organisaient des soirées culturelles. Abusz Werber lança dès son arrivée en Belgique, un journal destiné aux jeunes du Yung Bor, Unzer Rouf (Notre Appel), et publia deux brochures sur les thèses du parti. Il contribua aussi de sa plume à l'organe des ouvriers du LPZ en yiddish, Arbeter Tzaytung (Journal des Ouvriers), publié à Varsovie et diffusé dans toute l'Europe. Il envoya régulièrement des analyses politiques au mensuel du LPZ, Arbeter Vort (Voix ouvrière), diffusé en Europe occidentale et dont Yéhuda Tiberg était le rédacteur en chef.

Le choix de la langue était donc intimement lié au fait que le LPZ voyait dans la classe ouvrière juive l'agent moteur de l'histoire, la force sociale destinée à promouvoir le projet socialiste et national. Le second numéro du Unzer Wort clandestin de mars 1942, consacré à l'historique du LPZ, le clame haut et fort, dès sa première page : « Sans les masses juives desdits pays de la diaspora, la Palestine [...] ne pourra pas se construire et se développer, tandis que, faute d'un centre territorial en Palestine, les masses juives perdraient leur aptitude à mener une existence nationale. »

Le positionnement du LPZ dans la défense des masses prolétaires juives paupérisées représentait déjà une constante des années 1930, qui allait se renforcer sous l'Occupation. La protection de la population 
juive sur son lieu de vie s'imposait, car, sans elle, pas d'existence nationale possible ni de révolution prolétarienne.

\section{0 : l'action du Jewish Labor Committee}

Avec l'invasion éclair de la Belgique par la Wehrmacht le 10 mai 1940, la communauté juive se dispersa et prit la route de l'exode. Les familles Trocki-Werber, Tiberg, Hochberg et Tabakman se réfugièrent dans le sud-ouest de la France. Abusz et Shifra Werber avec leur fils Moiché (Michel), âgé de deux ans, ainsi que Dodé et Pauline et leurs parents se retrouvèrent à Revel, aux environs de Toulouse. Ils y séjournèrent de cinq à six mois.

Durant cette période, les leaders du LPZ reçurent du Comité ouvrier juif (Jewish Labor Committee) de New York des visas d'émigration pour les États-Unis. Yéhuda Tiberg et Shlomo Hochberg décidèrent de profiter de l'occasion et réussirent à rejoindre les USA. Kuba Costel, Chaïm Monek et Albert Blumenfrucht d'Anvers, émigrèrent en Eretz-Israel. Par la suite, Kubowitzki fut envoyé par les dirigeants du Yichouv en mission à New York où il séjourna pendant toute la durée de la guerre ${ }^{16}$.

Le Jewish Labor Committee (JLC) avait été créé à New York en 1934 par des dirigeants syndicaux affiliés au Bund et autres partis juifs de gauche, dans le but de soutenir les mouvements ouvriers d'Europe en proie à la répression nazie et fasciste ${ }^{17}$. Dès le début des hostilités, ils établirent une liste de leaders politiques particulièrement menacés et recherchés par les nazis, dont de nombreux membres du Bund et du Poalé Zion de gauche. Soutenus par l'American Federation of Labor, ils obtinrent l'accord de l'administration américaine pour attribuer des visas d'urgence «de visiteurs temporaires » aux personnes mentionnées ainsi qu'à leurs familles, en dehors des restrictions stipulées par les

\footnotetext{
16 Voir A. Weiss, «La vie et activité de Léon Kubovi en Belgique », Yédiot Yad Vashem, n ${ }^{\circ} 37$, février 1967, p. 6 (brochure publiée après sa mort en hébreu). Voir également : J.-Ph. Schreiber, « Kubowitzki Léon », dans Dictionnaire biographique..., op. cit., Bruxelles, 2002, pp. 204-205.

17 Voir C. Collomp, « The Jewish Labor Committee, American Labor and the Rescue of European Socialists, 1934-1941 », dans International Labor and Working-Class History, 68, 2005, pp. 112-133. Il est possible que Yéhuda Tiberg ait fait le choix d'émigrer parce qu'il était trop connu en tant que dirigeant du LPZ.
} 
quotas d'immigration officiels. Le JLC dépêcha son délégué, Frank Bohn, à Marseille en août 1940. Ce dernier prospecta dans les camps et centres de réfugiés du sud-ouest de la France et installa une antenne à Toulouse. Il travailla en étroite collaboration avec Varian Fry, l'émissaire du Centre américain de secours (Emergency Rescue Committee) chargé de sauver des intellectuels, artistes et hommes de science en danger. Après le départ de Bohn au mois d'octobre, Varian Fry poursuivit sa mission et traita directement avec le JLC des cas de personnes pour lesquelles il demandait des visas. L'initiative du JLC permit le sauvetage d'environ un millier de personnes. En 1995, Varian Fry fut honoré par Yad Vashem du titre de Juste parmi les Nations, pour avoir secouru de nombreux Juifs. Mais les Trocki et les Werber rejetèrent l'offre de visas d'émigration et rentrèrent en Belgique ${ }^{18}$. Sous leur égide, le LPZ fut la seule formation politique de la mouvance sioniste d'avant-guerre qui reprit ses activités au sein de la communauté immigrée dès le début de l'occupation et la poursuivit jusqu'à la Libération.

\section{« De si braves et bons enfants, tellement dévoués »}

Dans une lettre du 4 avril 1944, adressée à son ami Oscar (Ozer) Schwarz, membre du LPZ de France réfugié en Suisse, Werber rappelle certaines des raisons qui l'avaient amené à retourner en Belgique. Envoyée via messager clandestin, elle est formulée en termes codés pour tromper la censure. Werber y écrit : « ... Nous ne regrettons pas d'être ici, bien au contraire, nous en sommes très contents. Nous n'avons pas pu - et nous ne le pourrions jamais - abandonner nos vieux parents, des gens faibles et malades, pour améliorer notre existence. C'est un cas de conscience ! Plus que ça : un devoir familial, un devoir humain tout court! Le service que nous avons rendu, et continuons à rendre à l'oncle Yichouv [la communauté], à la tante Kibouzeinu [notre collectivité] et à la famille Merem [contraction de Moshé (et) Erem, dirigeant du LPZ en Palestine] nous donne une telle satisfaction, que nous sommes, malgré les quelques privations, très contents de nousmêmes... Surtout que j'ai de si braves et bons enfants, tellement dévoués, qu'il n'y a rien de difficile pour eux. \} ^ { 1 9 }

\footnotetext{
18 Voir M. Werber, op. cit., p. 21.

19 Yad Tabenkin Archives / YTA, Ramat Efal (Israël), 15-1/ 1-3, p. 2.
} 
Il est clair que cette lettre est l'expression d'un regard rétrospectif puisqu'en 1940, son auteur, Abusz Werber, ne pouvait pas connaître le tour qu'allaient prendre les évènements jusqu'en 1944. Mais elle fournit quelques éléments sur les raisons de son retour en Belgique. Elle reflète son sentiment de responsabilité à l'égard de ses coreligionnaires et l'impossibilité de les abandonner à leur triste sort, au moment tragique où s'installait l'occupation allemande. Se tenir à leurs côtés découlait d'une obligation d'ordre moral, « un devoir familial, un devoir humain tout court », à soulager les souffrances « des gens faibles et malades » et à assumer le sort de «nos vieux parents », soit les personnes les plus fragilisées face à la situation. L'expression « nos vieux parents » est à prendre au sens propre autant qu'au figuré puisque les deux familles Werber et Trocki avaient aussi les vieux parents de Shifra et Dodé à leur charge. Cette lettre met surtout en valeur, à travers l'allégorie du chef de famille désignant le chef du LPZ clandestin, la prise en charge, par ordre d'importance, d'abord de « l'oncle Yichouv » (la communauté juive tout entière), ensuite de « Kiboutzeinu » (le cercle plus restreint de la collectivité sioniste engagée) et, finalement, de « la famille Merem », les militants du LPZ. L’allégorie rend compte de la cohésion et des liens serrés entre ces « si braves et bons enfants », les résistants du parti entre eux mais alliés à d'autres partisans du sionisme, et de leur dévouement à l'égard des autres : non seulement les proches du parti, mais aussi la grande famille de la communauté dans sa totalité. Cette sollicitude des membres du LPZ à l'égard de la population juive persécutée, sans distinction d'appartenance politique, est aussi exprimée dans un rapport du $1^{\text {er }}$ mars 1945 sur l'activité du PZLPZ, avant et directement après la Libération. Selon ce rapport, « le parti n'a pas, pendant tout le temps de cette occupation brutale, abandonné les masses juives à elles-mêmes; il les a aidées matériellement et moralement et a défendu la dignité juive $»^{20}$.

\section{Retour à "la normale"}

Dès leur retour en Belgique en septembre 1940, les premiers rentrés du LPZ s'attelèrent à la restructuration du parti. Werber prit la relève

${ }^{20}$ YVS, Fonds Werber, O.29.2/4, p. 55. 
de Yéhuda Tiberg dont le départ le propulsa au premier rang du leadership. Il remit immédiatement sur pied la bibliothèque yiddish. L'école complémentaire Shul un Derziung reprit ses cours. Son personnel s'enrichit de nombreux membres de professions libérales radiés de leur poste suite aux mesures antijuives. Baruch Maizel, médecin renvoyé de son hôpital (suite à l'ordonnance de juin 1942 à l'exercice de la profession médicale) et reconverti dans l'enseignement, y tint la fonction de directeur pédagogique. L'écrivain Y. B. Zipper intégra l'équipe des enseignants, dont faisait déjà partie Shifra Werber. Charles Grabiner, un ancien du Dror proche collaborateur de Léon Kubowitzki dans les années 1930, en devint secrétaire. Un peu plus tard, une école similaire, dirigée par Faivel Blank, fut créée à Saint-Gilles pour éviter que les enfants n'aient à se déplacer. Ces deux écoles s'adaptèrent pour accueillir un plus grand nombre d'élèves quand, en décembre 1941, les enfants juifs furent exclus du système scolaire belge. Le mouvement de jeunesse du parti, le Yung Bor reprit ses activités. Meyer Tabakman, une fois libéré d'une période d'internement au camp d'Agde, dans le sud-ouest de la France, réanima le club sportif du LPZ, le Shtern. Dès son retour, il fut arrêté et subit plusieurs interrogatoires corsés au siège de la Gestapo, parce qu'il était connu comme l'un des leaders du LPZ d'avant-guerre ${ }^{21}$.

La sous-section juive du syndicat des maroquiniers, dominée avantguerre par le LPZ, réussit aussi à se restructurer dès les débuts de l'Occupation. Elle était dirigée par Abraham Domb, représentant du Secours mutuel, l'organe d'entraide du LPZ, et par Jacob Gutfrajnd de Solidarité juive, l'organe d'entraide animé par des Juifs affiliés au parti communiste ou sympathisants communistes. Cet exemple illustre la coopération entre LPZ et communistes, déjà établie bien avant l'été 1942. La rubrique que le Unzer Wort de mars 1942 consacre aux ouvriers maroquiniers souligne que la section juive du syndicat regroupait à cette date une centaine de membres et appelait tous les ouvriers du secteur à s’y rallier. Au printemps 1942, quand les Allemands émi-

\footnotetext{
21 Sur la restructuration de la communauté juive de Belgique sous l'Occupation, voir J.-Ph. Schreiber - R. Van Doorslaer (éds.), Les Curateurs du Ghetto. L'Association des Juifs en Belgique, Bruxelles, 2004 ; et plus particulièrement sur l'adaptation de la communauté au décret sur l'exclusion des enfants juifs des écoles publiques, voir dans ce même ouvrage B. Dickschen, « L'AJB et l'enseignement », pp. 233-260.
} 
rent les ordonnances excluant les Juifs de la vie économique et leur mise au travail obligatoire, les membres du syndicat se mobilisèrent pour obtenir l'indemnisation des ouvriers renvoyés des entreprises et celle des façonniers réduits au chômage ${ }^{22}$.

Le quatrième numéro de Unzer Wort, en juin 1942, rappelle à nouveau l'activité du syndicat de la maroquinerie et mentionne que sa direction se préoccupe du maintien des taux de salaire des ouvriers pour qu'ils puissent affronter l'augmentation du coût de la vie. Il informe aussi qu'à l'occasion du $1^{\text {er }}$ mai s'est tenue une réunion coorganisée avec le syndicat des tailleurs, où la présence des ouvriers maroquiniers syndiqués « était presque de cent pour cent ». Les problèmes des prolétariats juif et international y furent analysés. Gutfraynd souligne dans son témoignage qu'il était « le représentant de la section des maroquiniers dans le comité qui engagea le combat contre le travail pour l'occupant $\aleph^{23}$.

Gutfraynd fait ici référence aux appels, communs aux communistes et au LPZ, qui exhortaient les ouvriers juifs à s'opposer à la collaboration économique avec l'occupant. Ils encourageaient les artisans à refuser de travailler dans les ateliers et entreprises produisant pour le Reich et l'économie de guerre allemande. Pour ce faire, il fallait proposer une alternative et assurer un soutien aux réfractaires du travail. En juin-juillet 1942, les deux partis incitaient aussi à désobéir aux ordres de «prestation de travail » dans les camps de l'Organisation Todt du nord de la France, où les hommes furent assignés aux travaux de construction du Mur de l'Atlantique et, un mois plus tard, à la mise au travail obligatoire, qui devint synonyme de déportation vers l'Est. Si la lutte de la sous-section juive des maroquiniers conserva au premier stade son caractère syndical, "c'est dans ce combat qu'allait mûrir

22 Il faut signaler que les Werber-Trocki jouissaient d'une certaine sécurité économique. À ce stade, Werber fut forcé de liquider son atelier de fabrication de chaussures et se retrouva officiellement sans emploi. Mais, couvert par un homme de paille, il put continuer à gérer son affaire jusqu'à la Libération et ainsi assurer son indépendance économique. Dodé Trocki travaillait comme ingénieur pour une industrie protégée, les entreprises de gaz Nestor-Martin, et Pauline, sa femme, qui était médecin-dentiste, a continué à pratiquer dans le cadre de la communauté.

23 Témoignage de Jacob Gutfraynd, dans Partisans Armés Juifs. 38 Témoignages, Bruxelles, 1991, p. 175. 
l'idée de créer le premier groupe de partisans juifs », ajoute Gutfraynd ${ }^{24}$.

\section{Le Secours mutuel « Kegenzamtike Hilf »}

Le Secours mutuel, Kegenzamtike Hilf, l'institution d'entraide sociale du LPZ, fut initié par Abusz Werber en septembre 1940, dès son retour de l'exode. Elle s'érigea d'abord en caisse de solidarité qui fournit des prêts ou des subsides aux membres du parti en difficulté et à leurs familles. Le financement du Secours mutuel était assuré, à ses débuts, par les cotisations des adhérents et des donations de gens aisés. Un rapport d'activité du Secours mutuel, écrit en novembre 1944 par Werber afin d'obtenir un budget de fonctionnement, mentionne que le Secours mutuel a lancé sa première action en 1940, avec l'envoi de subsides aux Juifs de Belgique internés dans les camps du sud de la France, grâce auxquels ces derniers purent obtenir leur libération et retourner chez eux ${ }^{25}$. La seconde grande action du Secours mutuel, signalée dans ce rapport, s'est déroulée dans le contexte des premières déportations des Juifs de Belgique, en juin-juillet 1942, vers les camps du nord de la France. Malgré les mots d'ordre lancés par le LPZ pour les dissuader de se soumettre à « la prestation de travail», un grand nombre de requis se présentèrent quand même. Le Secours mutuel les munit de colis, de vivres et de vêtements de travail. Ceux qui étaient dépourvus de ressources reçurent une aide financière, consistant en l'attribution de pécules qui variaient de 300 à 800 francs belges de l'époque, ou de prêts remboursables à taux minimes. L'aide apportée par l'organisme d'entraide du LPZ consista ensuite à trouver des placements et « des couvertures » pour les réfractaires en Belgique. Meyer Tabakman s'investit particulièrement dans la recherche d'emplois dans l'agriculture, chez des paysans. Cette démarche eut un certain succès pour les femmes qui purent s'engager chez des particuliers comme employées de maison, domestiques et gardiennes d'enfants.

\footnotetext{
24 Ibid.

25 Rapport d'activité du Secours mutuel, écrit en novembre 1944 par Abusz Werber afin d'obtenir un budget de fonctionnement, YVS, Fonds Werber, 0.29.2/4, pp. 43-45. Ce rapport fut apparemment envoyé en Suisse soit au Congrès juif mondial soit au Joint (ou aux deux) pour justifier la demande de fonds.
} 
Le Secours mutuel a fonctionné en coopération avec Solidarité juive, dans le cadre de l'institution d'aide sociale de la communauté, Ezra ou OCIS (l'Cuvre centrale israélite de Secours), dès 1940. Srul Tabakman, l'un des piliers du Secours mutuel, mentionne dans ses mémoires que, dès le début de l'Occupation, il se retrouvait presque quotidiennement avec les membres de Solidarité juive à la cantine de l'Ezra autour d'une assiette de soupe chaude ${ }^{26}$. Le Secours mutuel n'était donc pas seul à utiliser l'infrastructure de services sociaux officiels pour étendre ses soins à la communauté toute entière. La coopération de ces deux institutions, le Secours mutuel et Solidarité juive, se solda par leur alliance pour constituer l'armature sociale du Comité de Défense des Juifs clandestin.

\section{Une fédération entre le LPZ, PZ et Poalé-Zion/Zéiré-Zion}

Le LPZ fédéra en son sein des militants de tendances diverses. Dès 1940, Werber œuvra au ralliement des anciens sionistes généraux, leurs leaders ayant émigré ou plongé dans la clandestinité. Parmi eux, Fela Liwer-Perelman et Charles Grabiner, qui avaient travaillé avantguerre avec Léon Kubowitzki, le représentant du sionisme dominant, apportèrent une contribution majeure au Secours mutuel. Fela LiwerPerelman en prit la direction dès le début des grandes rafles. Venu d'un autre milieu, Léopold Flam, philosophe et ancien membre du parti communiste, rejoignit l'équipe du LPZ de Bruxelles, après avoir quitté Anvers. Georges Goriely, proche des trotskistes, se rapprocha aussi du LPZ. Jusqu'à la fin de l'Occupation, Werber continua à unifier toutes les bonnes volontés et à intégrer les militants potentiels au travail clandestin. Si ses tentatives de rallier les membres de l'Hashomer Hatzaïr se heurtèrent à un refus, il réussit, en 1943, à recontacter des anciens du Dror. Une petite dizaine d'entre eux, regroupés autour de Icek Szatan et Szic Bibrovski relancèrent alors le Poalé-Zion/Zéiré-Zion et le Héchaloutz, mouvement de jeunesse pionnière. Le 29 avril 1944, Szatan écrivait à un ami de Suisse : « Vous n'ignorez pas sans doute qu'en 1942, tous nos jeunes amis sont allés rejoindre Marc, et que depuis il a

${ }^{26}$ I. Tabakman, Meiné iberlebung, Tel Aviv, 1957, p. 80 (en yiddish). 
fallu déployer de grands efforts pour regrouper les jeunes éparpillés dans le pays. $»^{27}$

Tout comme une partie de l'Hashomer Hatzaïr, les membres du Dror qui avaient échappé aux premières rafles, tentèrent de « rejoindre Marc », c'est-à-dire de se réfugier en Suisse où s'était replié Marc Jarblum, leader du PZ de France proche de Léon Blum ${ }^{28}$. Werber intégra ceux qui étaient restés en Belgique au travail social mené par le Secours mutuel. Dans cette même lettre, Szatan signale aussi que : «Zerubavel [Werber], qui a gardé de nombreux amis, avait commencé ce même travail longtemps avant nous. Il nous a proposé la collaboration au sein du Secours mutuel qu'il avait formé... Au bout de cinq mois... Sur les 500 familles aidées par le secours mutuel, plus de 200 sont assistées par nos soins $»^{29}$.

Le LPZ regroupa donc de fait une coalition sioniste unifiée des anciens du PZ mais aussi la jeune garde du Poalé Zion/Zéiré Zion, bien que ces derniers aient tenu à garder leur indépendance : en langage codé, on les nommait « la bande à Chat [Szatan] », qui se différenciait de « la bande à Ver [Werber] ${ }^{30}$. Pour eux comme pour les autres, les enjeux du sauvetage étaient aussi de « reconquérir les positions perdues durant notre absence de la vie publique ", afin de comptabiliser des points pour le contrôle de la communauté au lendemain de la Libération $^{31}$.

À cet effet, trois membres du Poalé-Zion/Zéiré-Zion, Nésanel Lewkowicz, Moïse Izgur et Icek Szatan, mirent sur pied l' « Office palestinien de Belgique », en liaison avec le Dr Chaïm Pozner, adjoint au chef du département de l'émigration de l'Agence juive, dont le siège se

${ }_{27}^{27}$ Lettre de Szatan à Lucien, YVS, Fonds Jarblum, P.7/38, p. 82.

${ }^{28}$ Président de la Fédération sioniste de France, Marc Jarblum s'était réfugié en Suisse en mars 1943. Il avait rejoint l'équipe du Congrès Juif mondial de Genève en tant que responsable de l'aide aux Juifs des pays d'Europe occidentale : la France, la Belgique, la Hollande et l'Italie.

${ }^{29}$ Lettre sans signature d'un ancien membre du Dror à Marc Jarblum en Suisse, le 30 avril 1944, YVS, Fonds Jarblum, P.7/38, p. 83. «Zerubavel » était le nom d'un des leaders du LPZ palestinien, utilisé ici, en langage codé, pour désigner Abusz Werber. 30 Jusqu'en fin 1943, le CDJ s'était appuyé sur les structures de Solidarité juive et du Secours mutuel. À cette date, les membres du Poalei-Zion/Zéiré-Zion commencèrent à se regrouper au sein du Secours populaire et contribuèrent à la distribution des secours via le CDJ.

31 Ibid, lettre à Marc Jarblum, le 30 avril 1944. 
trouvait à Genève. Ce dernier lança, en début 1943, l'opération des certificats d'échange germano-palestiniens, Austausch-Zertifikate, nommée aussi Sonder-Aktion des Palästina-Amtes, qui s'étendait à huit pays d'Europe ${ }^{32}$. Son objectif était d'obtenir pour « les vatikim », les vétérans sionistes de longue date de ces huit pays, le statut de candidat à l'émigration pour la Palestine qui leur attribuait la citoyenneté britannique. Cela les rendait éligibles à un éventuel échange germanopalestinien entre citoyens allemands détenus par les Britanniques et des Juifs vivant sous occupation allemande, possesseurs de ces certificats d'échange. Les négociations ne se faisaient pas « par le truchement de l'Association des Juifs en Belgique - AJB », comme le suggère Maxime Steinberg ${ }^{33}$. Elles impliquaient des tractations au niveau des sphères diplomatiques les plus élevées entre Berlin, Genève, Londres, Istanbul, Jérusalem, allant jusqu'à impliquer le Vatican. L'AJB n'avait aucun poids dans cette affaire. Au final, l'attribution de certificats d'émigration pour la Palestine ne se limita d'ailleurs pas aux seuls vétérans sionistes. Selon Daniel Dratwa, environ un millier de Juifs de Belgique profitèrent de cette option qui les sauva, dans de nombreux cas, de la déportation ${ }^{34}$.

\section{Un noyau dur de militants dévoués}

Ci-après se trouve la seconde liste des «vétérans sionistes » de Belgique transmise à Chaïm Pozner par Benjamin (Benno) Nykerk ${ }^{35}$, au

32 Rapport sur l'opération des certificats d'échange de Chaïm Barlas, délégué de l'Agence juive à Istanbul pendant la guerre, YVS, P.12-22, pp. 1-6. L'opération touchait « les vétérans sionistes » de la France, la Belgique, la Hollande, la Bulgarie et la Grèce, sous occupation allemande, mais aussi de l'Italie et la Hongrie que l'Allemagne n'avait pas encore envahies, ainsi que la Roumanie qui ne subit aucune occupation directe.

33 M. Steinberg, L'Étoile et le fusil. La Traque des Juifs, 1942-1944, tome III, vol. 1, Bruxelles, 1986, p. 195.

34 Voir D. Dratwa, « Nouveaux regards sur les listes d'échanges palestiniens », dans MuséOn, Revue d'art et d'histoire du Musée Juif de Belgique, 2, 2010, pp. 44-87.

35 Benjamin Nykerk était un industriel hollandais réfugié en Belgique. Juif de confession protestante, il s'était impliqué dans les affaires culturelles de la communauté dès 1940. Il devint trésorier du CDJ. En mai 1943, il fit un premier voyage en Suisse pour prendre contact avec les représentants des organisations juives "internationales" et aussi pour prospecter les routes de repli vers le havre de paix helvétique, le «Zwitser- 
cours de l'un de ses voyages en Suisse ${ }^{36}$. Jointe à une lettre envoyée le 26 octobre 1943 à Nathan Schwalb, le délégué du Héchaloutz en Suisse, Pozner lui demandait d'accélérer les démarches en leur faveur. Elle fut ensuite envoyée à Istanbul à Chaïm Barlas, représentant de l'Agence juive, qui la fit suivre à son siège central de Jérusalem pour entamer les procédures d'émigration auprès des autorités britanniques. Une fois ratifié, le nom du candidat à l'émigration était transmis à la légation du ministère des Affaires étrangères allemand en Suisse ainsi qu'au Comité international de la Croix-Rouge internationale, pour finalement aboutir en Belgique.

Cette liste est intéressante parce qu'elle contient 26 noms des principaux militants du LPZ-PZ candidats aux échanges germanopalestiniens, parfois sans avoir été consultés sur la question. Ne sont mentionnés que les noms des chefs de famille sans leur conjoint, ce qui fait passer à une cinquantaine les membres du noyau dur des militants du PZ-LPZ de Belgique pendant la guerre.

Parmi les noms cités dans la liste se trouve celui d'Avraham Domb, qui dirigeait la section juive du syndicat des maroquiniers avec Jacob Gutfraynd. Il était l'un des piliers du Secours mutuel et fera plusieurs voyages en France pour prospecter les possibilités d'évasion vers l'Espagne. Il participa à diverses opérations contre l'envahisseur. Gisèle, sa femme, fut activement impliquée dans le sauvetage d'enfants juifs.

Joseph Epsztejn était chargé, avec Elie Wajsglus, Hillel Kreitzman et M. Zilberszag, d'imprimer Unzer Wort ainsi que Le Flambeau,

weg », pour le compte du réseau d'évasion « Dutch-Paris ». Il en revint muni de lettres de crédit garantissant le remboursement par le Joint de prêts bancaires destinés à couvrir les activités de sauvetage du CDJ, ce qui permit le déblocage de fonds à cet effet. Fin 1943, le CDJ chargea Nykerk d'une nouvelle mission en Suisse (sa troisième apparemment). David Ferdman le remplaça à la trésorerie du CDJ. Mais repéré par la Gestapo, Nykerk dut "se mettre au vert" et se réfugier en France. Il fut arrêté à Paris, le 30 mars 1944. Déporté, il périt dans les camps (voir M. Steinberg, op. cit., pp. 106, 114 et 180-182 et notice « Benjamin Maurits Nykerk » dans J.-Ph. Schreiber, Dictionnaire biographique..., op. cit., p. 262).

36 Archives du mouvement travailliste, Institut Pinhas Lavon (Tel Aviv) / IPL, III37A- 2- 25. Cette seconde liste manquait à la collection réunie par Daniel Dratwa, op. cit. 
l'organe clandestin du CDJ en français. Avraham Riba (il manque sur la liste) secondait Abusz Werber à la rédaction de ces deux journaux.

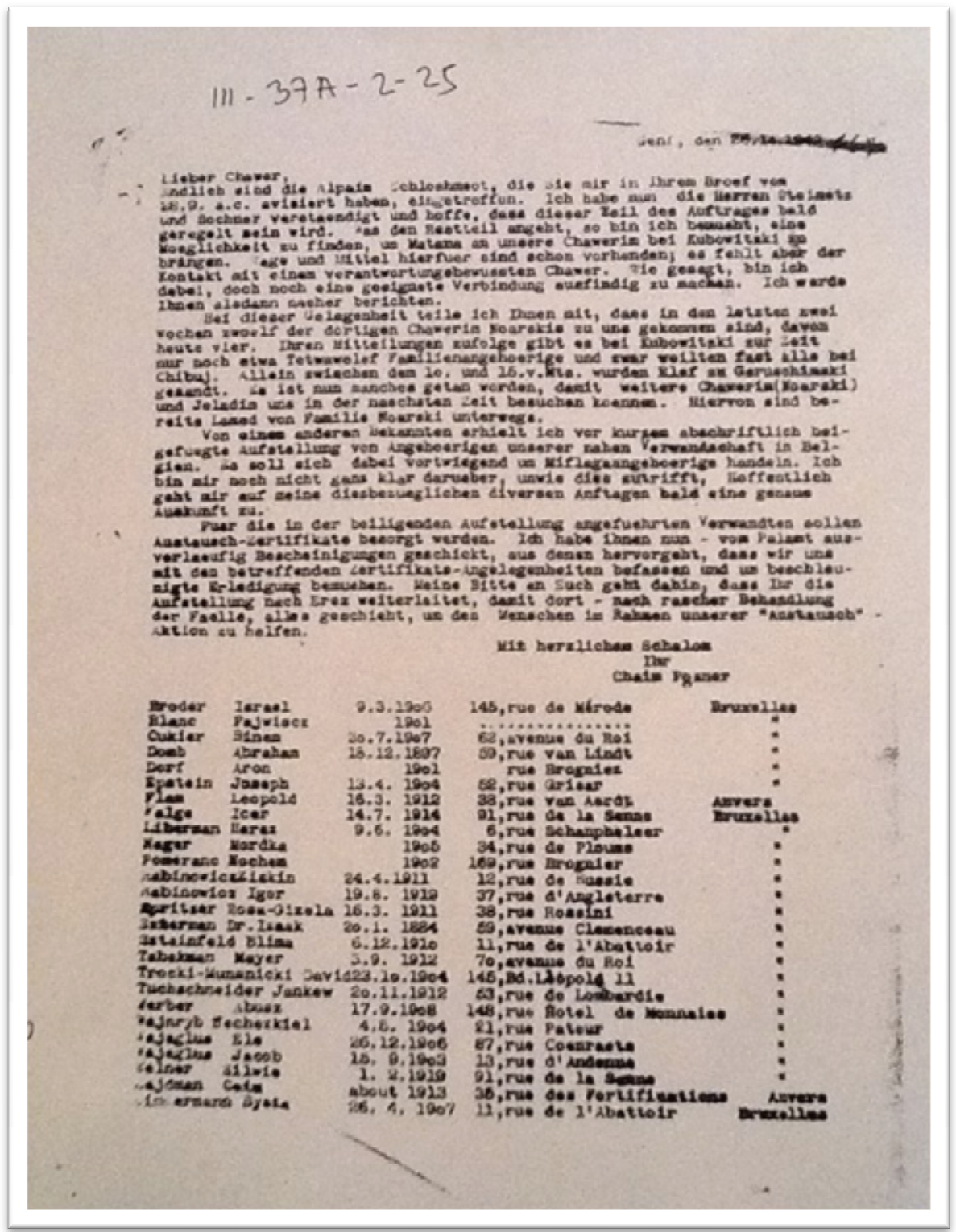


Léopold Flam fut à l'origine de la création de l'un des trois Comités de Défense des Juifs qui existèrent à Anvers ${ }^{37}$. Il lança la publication clandestine du CDJ en flamand, De Vrije Gedachte. Julia, sa femme, convoya et cacha des enfants juifs en danger. Elle entretenait la liaison entre le parti et Léon Platteau, l'adjoint au Secrétaire général du Ministère de la Justice, qui attribua au LPZ des subsides destinés à être distribués par le Secours mutuel aux personnes cachées. Ce fut grâce à l'intervention de « M. P. », Léon Platteau, que Julia obtint la libération de son mari du camp de Malines après sa première arrestation ${ }^{38}$.

Malka et Nahum Pomeranc, qui avaient habité avant-guerre à La Louvière, y avaient créé un groupe affilié au LPZ. Ils y avaient noué de nombreuses relations avec leurs voisins belges qu'ils approchèrent au temps des rafles. Certains répondirent positivement à leurs demandes et acceptèrent de cacher des enfants juifs.

Meyer Tabakman, l'animateur du Shtern, plaça des jeunes à la campagne qui pouvaient ainsi effectuer leur prestation de travail en Belgique. Il s'efforça de trouver des logements clandestins pour les réfractaires. Il fut impliqué dans tous les aspects de la Résistance. Arrêté à trois reprises, il s'évada deux fois en sautant du train : du convoi XIX (enregistré sous le no 499) le 15.01.1943; du convoi XX (no 1381) le 19.4.1943, avant d'être emmené par le convoi XXIII (no 7) le 15.1.1944, ce qui lui fut fatal. Reizel Tabakman, son épouse, était liée d'une vive amitié avec madame Solvay, la femme du grand industriel, qui obtint de nombreuses adresses de familles belges prêtes à prendre en charge des enfants juifs. Madame Solvay trouva d'ailleurs une cachette pour la petite fille du couple Tabakman et employa la sœur de Reizel comme domestique. Grâce à madame Solvay, les homes et colonies de vacances que les entreprises de son mari géraient ouvrirent

\footnotetext{
37 L'historien de la Shoah à Anvers, Lieven Saerens, a signalé l'existence successive de trois différents Comités de Défense des Juifs : l'un fut créé par Josef Sterngold, un autre par Abraham Manaster et le dernier par Léopold Flam. Tous furent démantelés suite aux arrestations de leurs militants. Voir L. Saerens, Étrangers dans la cité. Anvers et ses Juifs (1880-1944), Bruxelles, 2005 (édition française), pp. 817-862.

38 Je remercie Henri Flam, le fils de Julia et Léopold Flam, pour m'avoir donné cette précision et $m$ 'avoir transmis le manuscrit en français du journal de son père.
} 
leurs portes à de nombreux enfants en danger ${ }^{39}$. Reizel recevait de Shifra Werber des enfants à convoyer à leurs cachettes. Arrêtée et déportée à Auschwitz, elle survécut.

Shifra Werber fut la déléguée du Secours mutuel auprès de la Commission Enfance du CDJ. Elle convoya et plaça elle-même de nombreux enfants. En 1943, elle fut intégrée « au bureau » extérieur de la Commission et chargée de créer un service de « récupération », afin d'encaisser les contributions du CDJ à l'entretien des enfants cachés auprès de leurs parents ou de leurs proches, s'ils étaient encore en vie et s'ils en avaient encore les moyens. Elle travaillait avec deux agents de liaison : Sylvie Tuchschneider-Zelner et Régina Iszbicka.

Shifra Werber fut aussi secondée dans le sauvetage et convoyage d'enfants par Jechezkiel Wajnryb. Quand elle le somma d'être plus vigilant, il lui déclara : "Je me considère comme un soldat mobilisé dans une guerre. ».Il fut arrêté, déporté et assassiné dans les camps ${ }^{40}$.

Le nom de David Trocki figure aussi sur cette liste. Il collabora étroitement à la rédaction de Unzer Wort. L'appartement du couple Trocki constituait le centre névralgique des activités du LPZ clandestin. Dodé et Pauline furent tous deux arrêtés et déportés; seule Pauline survécut. Chaïm Zajdman, le leader d'avant-guerre du LPZ d'Anvers, ainsi que sa femme Frida, faisaient aussi partie de ce noyau dur de militants.

Mais les noms de nombreux autres résistants du LPZ manquent sur cette liste : celui d'Israël Tabakman, par exemple. Il était chargé, pour le compte du Secours mutuel, de visiter les sanatoriums, les homes de personnes âgées, l'hôpital de l'AJB et de distribuer colis alimentaires, vestiaires et subsides. Il fut la source des informations publiées dans Unzer Wort sur ces institutions. Sa contribution au travail social dans le cadre de Ezra lui avait permis d'obtenir une carte de légitimation de l'AJB, sans jamais en faire partie. Il avait établi "un atelier" où il entreposait des colis qu'il envoyait aux nécessiteux. Secondé par Israël Broder, par ses filles Leitché et Frida et par Sarah Gelbart-Baglayter,

\footnotetext{
39 Voir le témoignage de Ziva (Sonia/Suzanne) Reiss-Tabakman, la fille de Meyer et Reizel Tabakman, YVS, O.3/13351 (en hébreu). Elle avait quatre ans en 1942 quand la famille Wilden la prit en charge jusqu'à la Libération.

40 Voir le témoignage de Shifra Werber, Université hébraïque de Jérusalem, Institut du Judaïsme contemporain, département de documentation orale, 11.11.1965.
} 
il a régulièrement visité et apporté les secours à pas moins de cent vingt familles cachées. Suite à la déportation de ses quatre fils, l'une de ses filles et deux de ses belles-filles, Tabakman père surmonta son malheur en s'investissant à fond dans le sauvetage ${ }^{41}$.

Manque aussi à la liste le nom de Fela Perelman qui créa les écoles gardiennes « Nos Petits » et mit en œuvre ses relations avec le bourgmestre d'Uccle, Jean Herinckx, pour ensuite aider à cacher les enfants. Dès le début des grandes rafles, elle prit la direction du Secours mutuel. Manque également le nom de Menahem Konkowski, qui s'occupa de faux papiers et sera le fondateur de la 9e Brigade affiliée au Mouvement national belge (MNB) dans laquelle s'engagèrent de nombreux membres du LPZ; celui d'Elias Schlesinger, d'Arnold Perlgericht et celui d'autres qui s'investirent dans l'aide sociale.

La majorité des membres du Secours mutuel avait déjà dépassé la trentaine. Ils avaient constitué des familles avec enfants qu'ils mirent d'abord en sécurité pour se consacrer au sauvetage des enfants des autres, avec une plus grande marge de liberté et de mobilité. Ils avaient aussi, pour la plupart, un long passé de militantisme. Leur engagement dans la clandestinité découlait de leur engagement d'avant-guerre. Ils se connaissaient de longue date et pouvaient compter sur une confiance réciproque essentielle dans le cadre du travail clandestin. Ces facteurs (l'âge, la responsabilité d'une famille, la maturité, et l'engagement politique préalable), expliquent leur choix de s'orienter vers la résistance civile. Ils formaient surtout une équipe motivée, animée d'un grand dynamisme.

Pendant toute la durée de l'Occupation, Werber, dans le rôle de coordinateur et fédérateur, maintint un contact régulier avec 70 à 80 militants du LPZ. La cohésion du groupe et la détermination de ses membres stimulèrent l'engagement de nombreux autres volontaires. Le LPZ avait toujours compensé sa marginalité face aux institutions du mouvement socialiste mondial et du sionisme dominant ainsi que son manque de ressources par l'engagement sans faille et la ferveur d'adhérents particulièrement déterminés. Cette caractéristique forgée par une éthique de dévouement et de sollicitude à l'égard de la classe ouvrière juive se retrouve aussi bien dans l'équipe réunie dans le ghetto

${ }^{41}$ I. Tabakman, op. cit., pp. 152-158. Seuls l'un de ses quatre fils et l'une de ses bellesfilles survécurent à la déportation. 
de Varsovie autour d'Emanuel Ringelblum, le fondateur du Secours mutuel (Aleynhilf) et des archives clandestines Oneg Shabbat, que dans celle animée à Paris par David Rapoport, le dirigeant du Dispensaire de la Mère et l'Enfant et de la Colonie scolaire réunis dans le Comité de la rue Amelot ou que chez les membres du LPZ du cercle d'Abusz Werber à Bruxelles ${ }^{42}$. Selon l'historien israélien Elkana Margalit, spécialiste de l'histoire du LPZ, ce parti rassemblait des adeptes adhérant à une conception du monde totale et messianique fondée sur les principes du marxisme-léninisme, de sorte que, de toute la mouvance sioniste, il fut le plus motivé par les impératifs du combat révolutionnaire ${ }^{43}$.

\section{Vers l'insoumission}

La guerre contre l'Union soviétique, envahie par la Wehrmacht en juin 1941, radicalise les positions des sionistes de gauche et marque un tournant décisif dans le saut vers l'insoumission et la dissidence. Dès ses premiers numéros, Unzer Wort exprime une foi sans faille en la victoire finale de l'Armée rouge sur le nazisme et l'avènement du socialisme à l'issue des combats. Le journal réfute la thèse des deux impérialismes : «La nation des ouvriers n'a pas et ne peut pas avoir de buts impérialistes» et glorifie «le combat hérö̈que» de son armée, «l'avant-garde de la liberté » « qui lutte si courageusement pour la sauvegarde des libertés conquises en $1917 »^{44}$.

Selon Unzer Wort, la guerre insuffle une poussée révolutionnaire au processus de longue durée amorcé depuis la Commune de Paris et la Révolution de 1917. Elle représente la phase ultime dans l'affrontement entre la bourgeoisie en lutte pour conserver son hégémonie et le prolétariat de la patrie du socialisme et des pays assujettis. Le journal véhicule effectivement le message que la société occidentale et son pro-

42 À propos d'Emanuel Ringelblum, voir l'excellente analyse de S. D. Kassow, Qui écrira notre histoire? Les archives secrètes du ghetto de Varsovie, Paris, 2013. Sur David Rapoport, voir L. Lazare, La résistance juive en France, Paris, 1987, pp. 52-53, 55-59, 169-172.

43 Voir E. Margalit, Anatomie d'une gauche. Le Poalé-Zion de gauche en Eretz-Israël, 1919-1946, Tel Aviv, 1976, p. 17 (en hébreu).

44 Unzer Wort, « Le début de la fin », décembre 1941, 1, p. 3. 
létariat ne pourront se contenter, au lendemain des combats, d'une simple restauration des anciennes structures sociales. La destruction engendrée par la guerre constitue le moment opportun pour une transformation radicale du régime en Europe, dans la foulée des victoires de l'Armée rouge et calquée sur le modèle soviétique : « À l'issue de ce conflit sanglant, un monde nouveau devra se constituer $»^{45}$. Le journal réaffirme encore en mai 1944 que « les masses ouvrières ont trop saigné durant cette guerre pour retourner, à la Libération, à l'abominable exploitation de l'homme par l'homme... Nous avançons vers un ordre mondial radicalement différent $»^{46}$. Pour le LPZ, la révolution mondiale était bien à l'ordre du jour.

Cela impliquait « le devoir le plus urgent du moment de soutenir le mouvement de libération qui se propage dans le pays et, malgré notre état d'exception, rendre ce mouvement le nôtre ${ }^{47}$. Les masses juives dont le sort s'était toujours lié à celui du prolétariat révolutionnaire international obtiendront leur libération dans la lutte commune avec la classe ouvrière et les forces progressistes de Belgique. Il en allait de leur survie. Le Unzer Wort de juin 1942 proclame à ce sujet que « la solidarité internationale et la lutte contre le fascisme ne sont pas devenues des objectifs purement programmatiques vides de sens, mais des principes d'autopréservation dont dépend notre survie immédiate ».

À propos de la lutte commune avec les Belges et le soutien inconditionnel à l'Union soviétique, les positions du LPZ rejoignaient celles du parti communiste belge, aussi bien dans la terminologie que dans la stratégie de front unifié dans le sabotage contre les forces d'occupation, en renfort à l'arrière des troupes de l'Armée rouge. Elles allaient dans le sens des appels lancés par le Comité juif antifasciste sur les ondes de Radio Moscou, le 24 août 1941 et le 24 mai 1942, qui exhortaient à l'unification des forces contre le nazisme. Ces positions du LPZ, dans la ligne de l'internationalisme ouvrier, démentent les qualificatifs péjora-

\footnotetext{
45 Unzer Wort, 2, mars 1942, p. 2.

46 Unzer Wort, 24, $1^{\text {er }}$ mai 1944.

47 Unzer Wort, 4, juin 1942, p. 4.
} 
tifs qui lui ont été attribués de «pseudo-communistes » ${ }^{48}$ ou celui de Unzer Wort comme « journal nationaliste juif »49.

Le LPZ soutenait ainsi totalement la stratégie du Front de l'Indépendance (FI) et appelait les Juifs à s'y joindre. La fondation du Front de l'Indépendance, mouvement de masse créé sous l'égide du parti communiste belge (PCB), dans le courant de l'année 1941, suite à l'invasion de l'URSS, visait à la création d'un large front national pour l'indépendance, à caractère pluraliste et populaire ${ }^{50}$. Replié dans l'illégalité depuis le 25 août 1941, le PCB se lança d'une part dans la lutte armée et, de l'autre, élargit sa stratégie de front interclassiste en vue de déployer un vaste mouvement d'opposition aux armées de l'Axe pour alléger le poids de la lutte du peuple soviétique et accélérer la libération. Il opta pour l'unification de toutes les forces, comprenant catholiques, libéraux et socialistes, et tous ceux qui, individuellement, voulaient combattre pour la reconquête de l'indépendance nationale. Il consolida son rôle fédérateur avec la création de comités de masse par secteur ou profession, chacun doté de son organe de propagande propre. C'est dans ce contexte d'unification des forces résistantes au nazisme qu'il faut situer le patronage que le FI fut prêt à apporter à la création d'une section juive qui allait devenir le Comité de Défense des Juifs (CDJ).

\section{La plongée dans la clandestinité}

Les décrets de l'occupant sur la création de l'AJB en novembre 1941 et sur l'expulsion des enfants juifs des écoles belges dans le courant du mois suivant exacerbèrent les relations des membres du LPZ face aux autorités et aux officiels de la communauté. Ils lancèrent les premiers appels à la désobéissance civile par voie de tracts et surtout par Unzer Wort, dès décembre 1941. Abusz Werber, David Trocki et Avraham Riba, ingénieur lui aussi, formèrent le comité de rédaction, qui rassemblait les articles de nombreux contributeurs. Unzer Wort fut

\footnotetext{
48 P. Broder, Des Juifs debout contre le nazisme, Bruxelles, 1994, p. 44.

${ }_{49}$ M. Steinberg, L'Étoile et le fusil, op. cit., tome III, vol. 1, op. cit., , p. 83 et vol. 2, p. 16.

50 J. Gotovitch, Du rouge au tricolore. Les communistes belges de 1939 à 1944, Bruxelles, 1992, p. 120.
} 
le premier et seul journal de langue yiddish qui circula au sein du public immigré, de décembre 1941 à mars 1943. À cette date, les communistes lancèrent leur propre organe clandestin en yiddish, Unzer Kampf. Ils en publièrent cinq numéros pendant toute la durée de l'Occupation, le dernier datant d'août 1944, juste avant la Libération. Le Bund fit aussi paraître quatre numéros de son journal en yiddish, Der Morgen (dont deux numéros ont été retrouvés ${ }^{51}$ ).

Le LPZ, par contre, fit preuve d'une persévérance à travers l'édition, outre les tracts, de 28 numéros de Unzer Wort ainsi que des suppléments, diffusés presque mensuellement, tout au long de l'Occupation, à l'exception de la période des grandes rafles de juillet 1942 à février 1943, qui ne vit que 3 numéros en 7 mois. La collection reconstituée après-guerre réunit 18 numéros retrouvés. Le journal se présentait sous forme de 5 à 6 pages ronéotypées recto verso, sur papier jaune, ce qui lui vaudra l'appellation de « presse jaune ». Shifra Werber, Baruch Maizel et Charles Grabiner, jusqu'à son arrestation en septembre 1943, le tapaient à la machine à écrire sur stencils. Une équipe technique s'occupait de son impression sur ronéo qui tournait de 500 à 1000 exemplaires par numéro. Chaque bulletin était composé de 6 à 10 rubriques informant sur l'évolution des combats, la politique internationale, le sort des communautés juives d'Europe et de Belgique, les institutions officielles telles l'AJB, Ezra, et leurs dépendances, Malines, la vie sous l'Occupation, etc. Le journal était d'abord vendu au prix d'un franc belge dans les établissements de la communauté et diffusé ensuite parmi les Juifs cachés par ceux qui leur apportaient aides et subsides. Ils se passaient aussi "sous le manteau".

Au-delà de la transmission de l'information, avec le journal, le LPZ se munit d'un outil de communication "de masse", qui donna à la rébellion sa dimension sociale de Résistance civile non armée, qui passait par les mots d'ordre suivants :

- Refuser de se soumettre aux instructions de l'AJB, refuser de payer les cotisations exigées par l'AJB, refuser de se présenter aux convocations de l'AJB.

- Refuser de retirer les enfants juifs des écoles belges tant qu'ils n'en sont pas expulsés.

51 YTA, 15-3/9-11. 
- Refuser de travailler pour les Allemands.

- Se cacher (cette injonction apparaît dès juin 1942).

Ces consignes préparèrent le terrain et formèrent les mentalités à l'éventualité d'une plongée dans la clandestinité. Il est difficile d'expliquer le mouvement d'insoumission qui se propagea au sein du public juif menacé d'arrestation, durant la période des rafles, sans une préparation mentale à laquelle le journal contribua.

\section{La Résistance civile non armée : le Comité de Défense des Juifs (CDJ)}

Au printemps 1942, Werber établit ses premiers contacts avec les Juifs communistes, par l'intermédiaire de Lucien Mérinfeld, membre du Parti. Ils ne donnèrent pas de suites immédiates. Il fallut attendre qu'Émile Hambresin et Hertz Jospa, délégués du PCB, reçoivent l'aval de leur direction nationale pour créer une Section de Défense des Juifs affiliée au Front de l'Indépendance ${ }^{52}$. En juin-juillet 1942, ces derniers relancèrent l'idée d'une alliance et s'adressèrent alors à Werber. Mais ce ne fut qu'à la mi-septembre 1942, durant ce que l'historien Maxime Steinberg appela « les cent jours de la déportation », que les échanges entre les deux parties, communiste et LPZ, aboutirent à la mise en place du CDJ et au lancement de son activité. Fin septembre 1942, Hambresin, Jospa et Werber obtinrent finalement l'adhésion d'un Chaïm Perelman hésitant. L’universitaire radié de l'ULB et membre de l'AJB, au poste d'adjoint au chef de son service social, accepta alors de s'engager dans la clandestinité. La participation de membres de l'AJB au CDJ fut le produit d'une initiative commune du LPZ et des communistes. Le mouvement de Résistance français, Combat, développa cette même stratégie de «noyautage des administrations publiques » (NAP), dont les objectifs étaient de recueillir les informa-

52 Émile Hambresin, journaliste démocrate-chrétien, n'était pas membre du parti communiste mais avait rejoint le FI. Il avait présidé avant-guerre la Ligue belge contre le Racisme et l'Antisémitisme. Hertz Jospa, ingénieur d'origine juive et communiste de longue date, avait milité à ses côtés. Leur contribution majeure fut d'avoir réussi à convaincre la direction du PCB de soutenir la création du CDJ. Jospa fut arrêté et déporté à Buchenwald en juin 1943. Il survécut. Hambresin fut arrêté et déporté un mois plus tard. Il périt dans les camps. 
tions, retourner l'institution à l'avantage de la Résistance et préparer la reconstruction.

Pour les communistes, dans le cas de la Belgique, le leader du LPZ constituait un interlocuteur privilégié : en tant que socialiste révolutionnaire, ses idées rejoignaient les leurs. Il était lié aux milieux ouvriers juifs et à "la rue juive" en général. Ses positions sionistes et ses dons de diplomate assurèrent le ralliement au CDJ de sionistes généraux, Perelman en particulier, pour lesquels accepter le patronage du Parti communiste n'allait pas de soi. Le LPZ put ainsi occuper une position charnière de parti-tampon qui lui permit de créer des passerelles entre communistes et sionistes de toutes tendances.

La coopération préalable entre le Secours mutuel et Solidarité juive, dans l'action syndicale et l'entraide sociale, avait préparé le terrain à l'entente entre communistes et LPZ. Ces deux structures, qui conserveront leur indépendance dans la concurrence, constitueront l'armature du CDJ. Malgré les antagonismes, l'union fut maintenue jusqu'à la Libération. La cohésion et le front unifié soudèrent les énergies qui se mobilisèrent dans le sauvetage des Juifs.

Les rafles commencèrent pourtant bien avant le lancement du CDJ. Shifra Werber, Fela Perelman, Julia Flam, Reizel Tabakman et d'autres avaient déjà caché de nombreux enfants avant la création de la Commission Enfance du CDJ. Elles en furent les initiatrices autant que les communistes. L'activité de la Commission Enfance se structura autour de ces deux piliers, avec la recherche d'abris pour les enfants, leur convoyage, le payement des pensions ainsi que leur suivi pour soins médicaux, vestiaire, et le changement de cachette en cas de nécessité urgente. Shifra Werber remplira d'ailleurs la fonction de déléguée du Secours mutuel auprès d'Yvonne Jospa, à la tête de la Commission Enfance. Son intégration au service de récupération en 1943 renforcera la structure binaire initiale de l'institution.

Il serait prétentieux de vouloir rendre compte de toutes les activités du CDJ, de la mi-septembre 1942 à 1944, dans le cadre de cet article. Nous nous contenterons de rappeler qu'Abusz Werber et Chaïm Perelman furent les deux seuls responsables du CDJ initial à avoir évité l'arrestation, de sorte qu'ils purent apporter à l'institution stabilité et continuité. Perelman, à la tête de la Commission d'aide aux adultes du CDJ, fut secondé par Werber, chargé du service des faux papiers, et 
par « Robert » (Israël Mandelbaum) ainsi que « Richard » (Icek Wolman), par la suite. Ces deux derniers étaient membres de Solidarité juive. La Commission Adultes du CDJ reproduisait done la structure binaire de la Commission Enfance avant de s'ouvrir à d'autres participants. La distribution des soins aux Juifs cachés nécessita l'engagement de nombreuses bonnes volontés qui apportèrent viatiques, subsides, faux papiers, tickets de ravitaillement, colis, vêtements, médicaments et journaux ${ }^{53}$. On ne manquera pas de rappeler la participation de nombreux non-Juifs au CDJ : à sa direction, celle d'Émile Hambresin en premier lieu, de Benno Nykerk, d'Yvonne Nevejan, directrice de l'CEuvre nationale de l'Enfance (ONE) qui apporta une contribution majeure au sauvetage des enfants, et du professeur Allard, aux côtés de nombreux coopérateurs au fonctionnement des commissions.

À l'automne 1943, le CDJ national (CNDJ) coordonna le sauvetage dans l'ensemble du pays. Y participèrent deux membres du LPZ, Léopold Flam et Abusz Werber, ainsi que deux communistes, Pierre Broder de Charleroi et Albert Wolf de Liège. La représentation nationale du CDJ reposait donc essentiellement sur ces deux partis, sans exclure d'autres apports qui allèrent en se multipliant. En dépit de ses critiques à l'égard du LPZ, Maxime Steinberg reconnaît que « jusqu'à la fin de 1943, le CDJ avait pris essentiellement appui sur les deux principales formations existant avant la déportation, Solidarité juive des communistes et le Secours mutuel des socialistes-sionistes de gauche $\aleph^{54}$.

\section{La presse clandestine du CDJ}

Werber fut par ailleurs le responsable de la Commission Information et Propagande du CDJ. Il lança, en mars 1943, la publication de

\footnotetext{
${ }^{53}$ Sur l'activité du CDJ, voir M. Steinberg, L'Étoile et le fusil. Les cent jours de la déportation des Juifs de Belgique, tome II, Bruxelles, 1984 et Id., L'Étoile et le fusil. La Traque des Juifs, 1942-1944, tome III, vol. 1, Bruxelles, 1986; R. De Lathouwer, liquidateur du CDJ au statut de la Résistance Civile, Témoignages et Documents recueillis entre 1947 et 1951, exemplaire YVS, O.29/46, p. 23 ; L. Steinberg, Le Comité de Défense des Juifs en Belgique 1942-1944, Bruxelles, 1973.

54 M. Steinberg, L'Étoile et le fusil. La Traque..., op. cit., tome III, vol. 1, p. 197.
} 
l'organe clandestin en français du CDJ, Le Flambeau, et Léopold Flam De Vrije Gedachte, son pendant néerlandophone. La contribution du LPZ aux publications clandestines du CDJ fut donc primordiale.

Le Flambeau sortit sept numéros, le premier datant de mars 1943 et le dernier de mai 1944. À l'exclusion des numéros 5 et 6 de janvier et février 1944 qui ont sans aucun doute été écrits par Flam, Le Flambeau reprend des articles ou des résumés d'articles déjà parus dans Unzer Wort. Une étude comparative des articles publiés dans ces deux journaux confirme ce constat. Comme le mentionne Srul Tabakman, Avraham Riba était chargé d'en faire la traduction ${ }^{55}$. Le Flambeau constitue de fait une version abrégée de Unzer Wort. Il s'adressait à la population belge en particulier et s'attachait à démonter la propagande antisémite officielle. Les sujets internes à la communauté, sur la Palestine et le monde juif, développés dans Unzer Wort, en étaient évacués. L'organe du CDJ en français diffusait des informations générales sur l'évolution du front et la scène internationale, mais mettait surtout l'accent sur les persécutions contre les Juifs. Il divulgua notamment l'information sur le sort des Juifs déportés en Pologne, incitant les Belges à les protéger. Son objectif déclaré était de renseigner la population belge «sur la guerre d'extermination sauvage menée par l'occupant nazi contre nous [les Juifs] ».

De Vrije Gedachte voulait aussi rallier la population belge de langue flamande au sauvetage des Juifs. Le journal sortit quatre à cinq numéros, dont la publication suivit les périodes d'incarcération de Léopold Flam à Malines. Les premiers De Vrije Gedachte virent le jour avant l'arrestation de ce dernier, en mai 1943. Il fut libéré en octobre, reprit immédiatement son activité clandestine et relança la rédaction de $D e$ Vrije Gedachte, dont les deux numéros de janvier et février 1944 sont identiques aux numéros 5 et 6 du Flambeau des mêmes dates. Ces quatre numéros furent imprimés chez le même imprimeur à Anvers ${ }^{56}$. Tous quatre sont donc très vraisemblablement de la plume de Flam. En mars 1944, Flam fut arrêté une seconde fois et déporté à Buchenwald. Il survécut, mais De Vrije Gedachte cessa d'exister avec sa déportation.

\footnotetext{
55 I. Tabakman, op. cit., p. 123.

56 Lettre d'Abusz Werber à Roger Van Praag du 28.11.1950, YVS, Fonds Werber, $0.29 .2 / 3$, p. 45.
} 


\section{Unzer Wort}

Les fonctions de Unzer Wort furent de loin bien plus vastes et plus complexes que celles des deux publications clandestines du CDJ. La contribution majeure de l'organe du LPZ fut de l'ordre de la résistance spirituelle : une arme qui insuffla aux Juifs humiliés le courage de surmonter l'adversité quotidienne. Ses révélations sur les desseins nazis constituèrent une donnée indispensable pour inciter les Juifs à se cacher et à prendre les précautions nécessaires à leur survie. Il informait sur l'ampleur et l'unicité de la catastrophe touchant les communautés juives d'Europe occupée. Même si l'état des connaissances transmises par le journal ne reflétait pas encore une prise de conscience exacte de la Shoah et de la « solution finale », il rendait compte de l'étendue de la destruction. Le journal lançait aussi les consignes de comportement à suivre : se cacher, redoubler de prudence, garder le moral, soutenir la Résistance belge, rejoindre les rangs des combattants. Les appels à la lutte armée apparaissent dès juin 1943: «Se cacher, c'est une très bonne chose ; toutefois cela ne peut pas constituer un but en soi, mais seulement le moyen permettant à mener le combat contre l'antisémitisme meurtrier des fascistes. Donc n'attendez plus, ouvriers et membres de la communauté juive... Prenez exemple du courage et du sacrifice de nos frères, combattants héroïques tombés à Varsovie... Rejoignez les rangs des combattants pour la Liberté ! » ${ }^{57}$.

Ces appels se font plus insistants à partir de juin 1944, quand le LPZ donne son aval à la création de la 9e Brigade affiliée au Mouvement national belge (MNB), sous la direction de Menahem Konkowski. Le journal rendait aussi régulièrement hommage aux résistants tombés dans la lutte contre l'occupant, en Belgique, apportant son sceau de légitimité à leurs actions.

Unzer Wort élève la voix “d'en bas" des Juifs, interdits de parole. Il recrée un espace de libre expression leur offrant une autoreprésentation alternative à celle de l'AJB. Le journal réactive les repères identitaires, ancrés dans la mémoire juive, dans celle du mouvement ouvrier international et de la communauté nationale afin de restituer aux persécutés et humiliés leur dignité humaine. Ces repères leur permettent

${ }^{57}$ Unzer Wort, 11, juin 1943. 
d'affronter les vicissitudes du présent avec le souvenir d'un passé glorieux et exemplaire. Les références au passé sont ranimées au travers d'articles consacrés aux dates commémoratives de la fondation du PZ et du LPZ, à Borochov, à l'écrivain yiddish Y. L. Peretz, aux côtés de commémorations de portée symbolique puisées dans le calendrier révolutionnaire international ou national, comme l'anniversaire de la Révolution d'Octobre, de la Commune de Paris, la célébration du $1^{\text {er }}$ Mai et du 11 novembre. Fidèle à la vocation du LPZ, Unzer Wort combine ainsi identités juive et socialiste.

En outre, le journal, tout comme les œuvres littéraires qu'il rappelle, rallie des individus isolés, souvent reclus et coupés du monde extérieur, autour d'un champ unifié d'informations, de représentations et de symboles, leur permettant de s'affilier à une communauté imaginée. Il fait la liaison entre les Juifs dispersés mais soumis à un destin commun. Comme l'a montré le sociologue Benedict Anderson, la presse écrite, le journal et le roman, producteurs d'images et de symboles partagés par des personnes inconnues l'une de l'autre, constituent un facteur important dans la formation de groupes sociaux ${ }^{58}$.

Les textes du journal assurent leurs lecteurs de la libération proche et de l'avènement d'un monde meilleur. Ils leur permettent de se projeter dans l'avenir autour d'un projet collectif : la construction du socialisme et la création d'un État juif indépendant. Ils s'efforcent de restituer aux Juifs le contrôle de leur histoire et de les rendre maîtres de leur destin afin de réduire leur sentiment d'impuissance. Surtout, à partir d'août 1943, date à laquelle le comité de rédaction de Unzer Wort reçut journaux et brochures envoyés de Suisse, les nouvelles en provenance des pays libres et en particulier de la Palestine les rattachent au monde extérieur. Les expressions de soutien et les manifestations en leur faveur les sortent de leur isolement.

Le journal devint ainsi un lieu d'échange virtuel en remplacement de l'espace communautaire aboli. De façon paradoxale, Unzer Wort s'érigea en vecteur de dialogue entre les officiels de l'AJB et ses contestataires du LPZ. Ses articles mentionnent les réactions des membres de l'AJB aux accusations portées contre eux, par voie du journal. Le $\mathbf{n}^{\circ} 9$ de mars 1943 signale que « l'entrefilet paru dans l'édition de février de

58 Voir B. Anderson, L'imaginaire national. Réflexions sur l'origine et l'essor du nationalisme, Paris, 2006. 
Unzer Wort, concernant les méthodes draconiennes d'Ezra lors de la distribution de son aide misérable, a suscité un intérêt particulier dans les cercles visés ${ }^{59}$. L'entrefilet en question s'insurge contre la réduction de moitié de l'aide accordée aux familles par le service social de l'AJB et son mode de fonctionnement stipulant que «plus grand sera le nombre de Schnorrers (mendiants) envoyés à Malines, moins d'argent on aura besoin pour leur entretien $»^{60}$.

Dans le $n^{\circ} 21$ de février 1944, on peut noter la mise au point adressée par la rédaction du journal aux « dirigeants de l'AJB [qui] ont réagi violemment » aux griefs émis dans le numéro précédent, les accusant d'avoir fourni au camp de Malines, à l'occasion de la nouvelle année, du champagne pour ses dirigeants et des clous et des barbelés pour clôturer les wagons de déportation. L'auteur de l'article répond aux justifications données par l'AJB à ce sujet. Le $\mathbf{n}^{\circ} 25$ du mois de mai suivant constate encore que « chaque fois que nous publions un article concernant les activités de l'AJB, c'est la commotion chez ces voyous ». Ce même numéro rappelle que l'AJB avait envoyé les convocations aux jeunes requis pour le travail forcé dans le nord de la France, en juin-juillet 1942 et celles « invitant » les Juifs à se présenter volontairement à Malines pour le départ vers l'Est, « signe de Caïn gravé sur... cette institution ». Il condamne aussi sa gestion dictatoriale selon « le Führer Princip. »

Le $n^{\circ} 26$ de fin juin 1944 indique à nouveau que « les directeurs de l'asile [pour vieillards] se sont offusqués de l'article publié sur leur comportement dans le Unzer Wort précédent [...] Nous ne pouvons pourtant pas passer sous silence de tels débordements »: soit les restrictions alimentaires et les coupures d'électricité injustifiées ainsi que la terreur et les punitions corporelles infligées par le personnel de l'hospice de la rue de la Glacière à ses hôtes.

Il est clair que les rédacteurs du journal étaient non seulement au courant de la gestion des institutions officielles de la communauté imposée mais qu'ils recevaient aussi des rapports provenant de "l'intérieur" sur ce qui se passait dans les coulisses de l'AJB. Ils étaient

\footnotetext{
59 La critique des méthodes de Ezra exprimée dans l'article «Intérêt et sécurité prédominent », Unzer Wort, 9, mars 1943, est reproduite dans sa totalité dans Michel Werber, op. cit., pp. 52-53.

60 Voir rubrique « L'île de désolation de Bruxelles », Unzer Wort, 8, février 1943.
} 
bien informés des réactions des institutionnels à leurs critiques. Le seul fait que ces derniers se sentaient surveillés de "l'extérieur" constitua un moyen de pression.

Le journal établit des passerelles qui permirent des échanges certes conflictuels - entre les officiels et la Résistance. Ce « dialogue par l'intimidation » eut pour effet, direct ou indirect, d'améliorer quelque peu les services sociaux rendus par l'AJB. En effet, en mai 1944, l'article de Unzer Wort sur la section préposée à l'assistance sociale admet que « l'attitude de Ezra et du service social du boulevard d'Anvers envers les demandeurs d'aide [...] est devenue plus humaine » «On a cessé de considérer les Juifs affamés comme des mendiants. » Ezra s'est réformée : les nécessiteux n'ont plus besoin de se présenter chaque semaine pour recevoir leurs misérables subsides. Une fois par mois suffit. La distribution à la cantine populaire se fait aussi de façon plus équitable. On peut lire, dans le même numéro, que l'attitude de ses employés «s'est humanisée et l'atmosphère interne s'est améliorée ». Selon toute probabilité, les menaces émises à l'égard des membres de l'AJB, par voie du journal, de comptes à rendre dans le futur, œuvrèrent en tant que force de dissuasion ${ }^{61}$. En outre, il ne faut pas oublier que les services sociaux du CDJ proposaient une alternative à ceux offerts par l'AJB, que les nécessiteux désertaient progressivement. De ce fait, en prenant la responsabilité du sort de toute la communauté, le CDJ s'imposa en direction contre-institutionnelle à l'AJB.

L'engagement du LPZ aux côtés des Juifs communistes, les deux piliers de l'entraide sociale clandestine, en faveur de leurs frères persécutés constitua un premier « écran protecteur » face à la déportation. À ce phénomène de sauvetage des Juifs par eux-mêmes, le patronage du parti communiste belge via le Front de l'Indépendance vint renforcer la protection autour des personnes menacées. Par un effet de boule de neige ou de « réactivité sociale », la cohésion du groupe des résistants et leur dévouement aux masses juives rallièrent des cercles de défense plus larges au sein de la population et de l'administration belges. Ils mirent ainsi de la distance entre les acteurs de la « solution

61 Voir Unzer Wort, 11, juin 1943 : « Ces gens de l'AJB [...] ne devront-ils pas rendre des comptes de la tragédie du sang ayant eu lieu entre les murs de l'Ezra »; et dans le no 17 , octobre 1943 : « en d'autres temps, nous rappellerons à ces messieurs... ». 
finale » et leurs victimes désignées, les Juifs. En accord avec « la théorie des trois écrans » développée par le sociologue Jacques Semelin dans son livre Sans armes face à Hitler, ces conditions de la Résistance civile non armée en Belgique assurèrent la survie de $55 \%$ des Juifs de Belgique, comme l'a montré Maxime Steinberg qui attribue au CDJ le sauvetage de 3.000 enfants et de 5.000 adultes $^{62}$. Durant les deux dernières années de l'Occupation, de nombreuses personnes purent donc être sauvées grâce à ce type de Résistance. En l'espace de deux mois, du 4 août 1942, départ du premier convoi vers Auschwitz, jusqu'au 31 septembre 1942, deux tiers de l'ensemble des victimes de la Shoah en Belgique, soit 17.000 Juifs, furent déportées... ${ }^{63}$

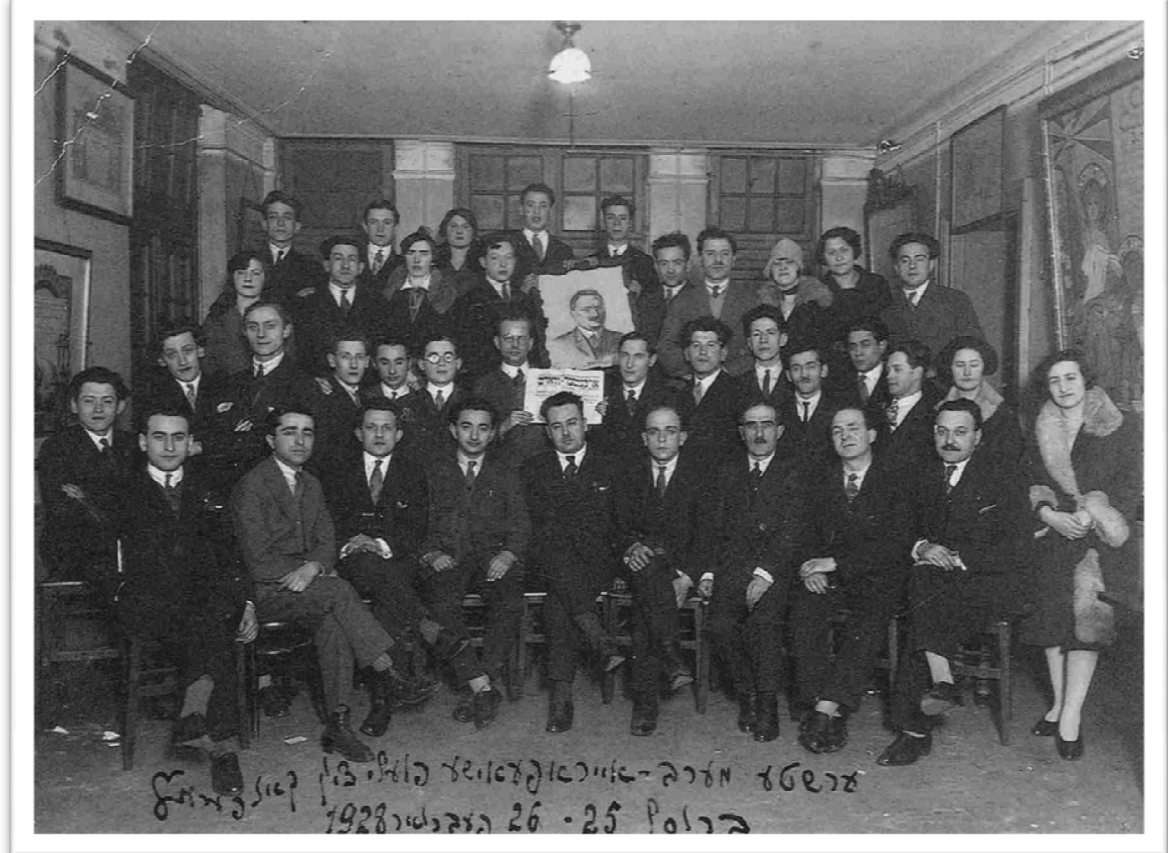

Le congrès des délégués du Linké Poalé Zion (LPZ) d'Europe occidentale à Bruxelles en 1928. David Trocki (debout, $2^{\mathrm{e}}$ rang, $4^{\mathrm{e}}$ de droite) et Yehuda Tiberg (assis, $1^{\mathrm{er}}$ rang, 4 e de droite) y représentent la Belgique. (C) Collection Yad Tabenkin

${ }^{62} \mathrm{~J}$. Semelin, Sans armes face à Hitler. La résistance civile en Europe 1939-1945, Paris, 1998 (édition de poche).

63 Maxime Steinberg, L'Étoile et le fusil. La Traque..., op. cit., tome III, vol. 1. 


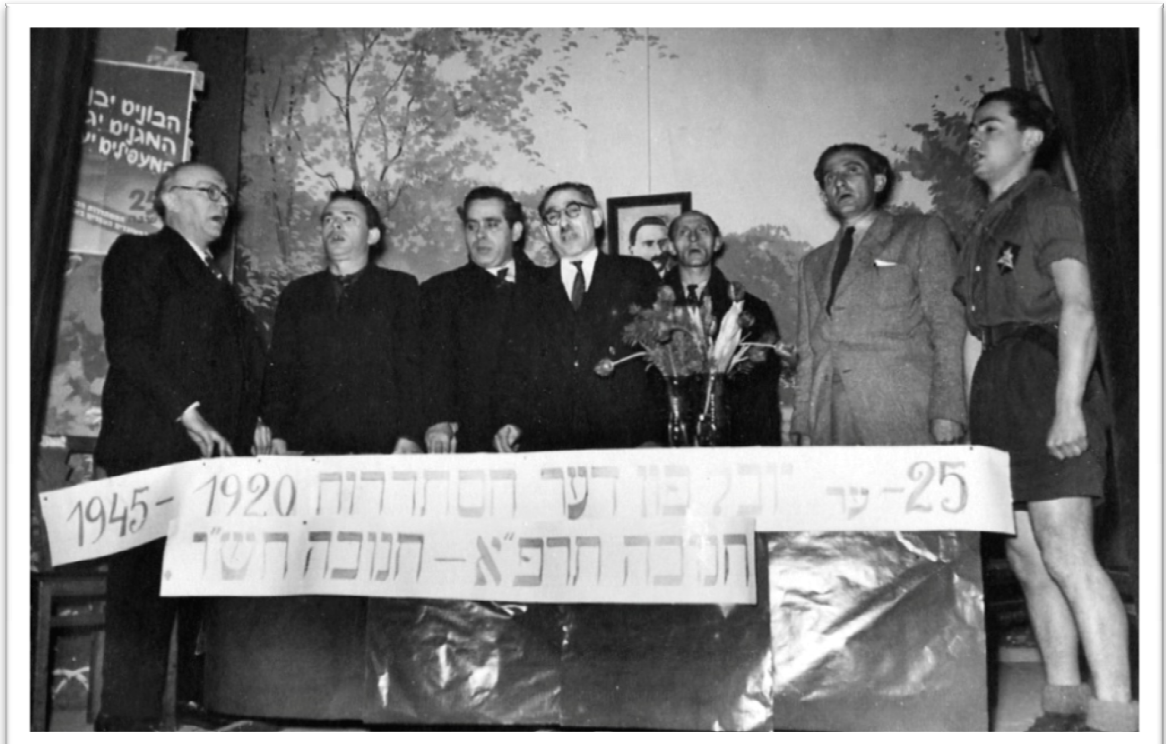

Le LPZ à la Libération : Abusz Werber (deuxième à droite) et Israel Tabakman (4e de droite). (C) Collection Yad Tabenkin

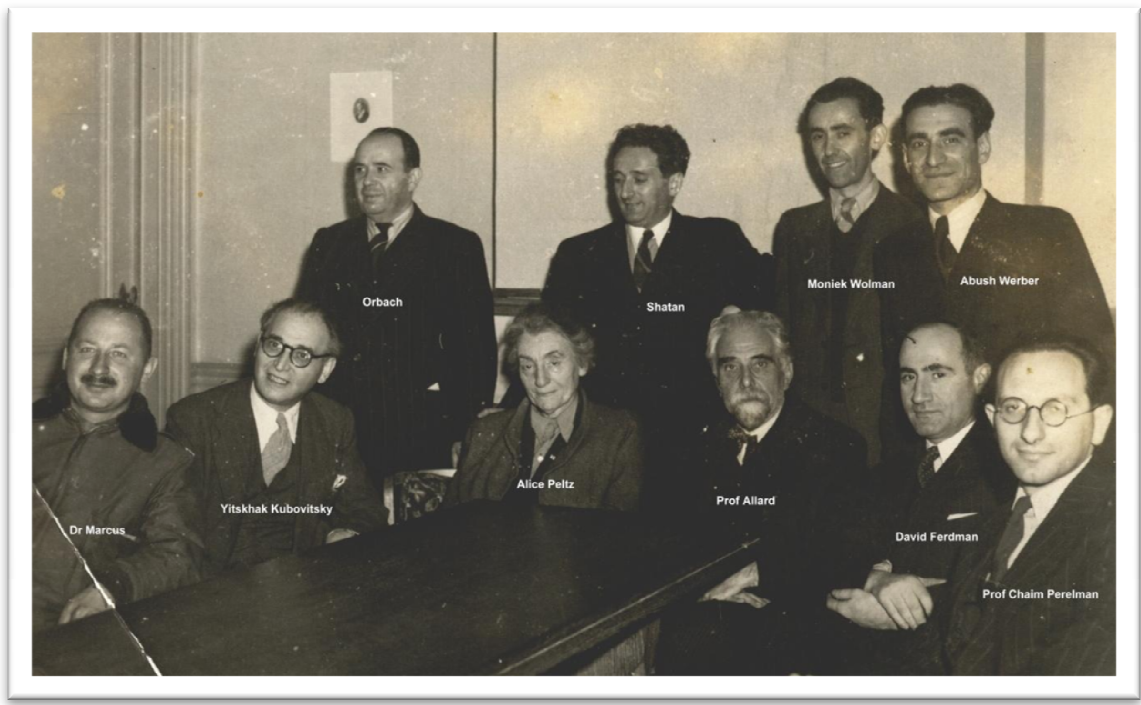

Le Comité de Défense des Juifs au lendemain de la Libération (C) Collection Yad Tabenkin 